\title{
Sperm Telomere Length in Men with Normal and Abnormal semen Parameters. A Pilot Study
}

\author{
Ewa Boniewska-Bernacka ${ }^{1 *}$, Anna Pańczyszyn ${ }^{1}$ and Natalia Cybulska ${ }^{2}$ \\ ${ }^{1}$ University of Opole, Poland \\ ${ }^{2}$ GMW-Embrio Opole Poland
}

*Corresponding author: Ewa Boniewska-Bernacka, University of Opole, Poland

\begin{abstract}
ARTICLE INFO
Received: 幽 August 30, 2019

Published: 幽 September 06, 2019

Citation: Ewa Boniewska-Bernacka, Anna Pańczyszyn, Natalia Cybulska. Sperm Telomere Length in Men with Normal and Abnormal semen Parameters. A Pilot Study. Biomed J Sci \& Tech Res 21(1)-2019. BJSTR. MS.ID.003559.
\end{abstract}

Keywords: Telomere; Biomarker; Semen; Male
ABSTRACT

Objective: Male infertility is a growing social problem. The measurement of telomere length may be a new marker that can help to assess reproductive age of males and assess the chances of in vitro fertilization.

Aim: The aim of our study was to investigate whether there are any differences between telomere length in men with normal and abnormal semen parameters.

Materials and Methods: Telomere length was analyzed in 17 patients with normal and 28 patients with abnormal semen parameters using the telomeric repeat amplification protocol and Quantitative Real-Time Polymerase Chain Reaction (qPCR).

Results: Mean sperm telomere length in all donors was $49 \pm 24 \mathrm{kbp}$ per haploid genome. However, patients with abnormal semen parameters had significantly longer telomeres compared with patients with normal semen parameters. Higher variability in sperm telomere length was observed in patients with abnormal semen parameters in comparison to patients with normozoospermia.

Conclusion: The results suggest that telomere length assessment may be a useful future marker that may help to assess sperm cell fertilization capacity.

\section{Introduction}

Fertility is a growing social problem that affects about $15 \%$ of couples [1]. Reduced quality of semen is a major problem in many cases. Standard diagnostic procedures based on semen parameters are often insufficient to explain the causes of male infertility, especially in men with idiopathic infertility [2]. Recent studies showed that telomere length assessment may be a promising biological marker of men reproductive age [3]. Telomeres are linear parts of chromosomes that protect chromosome ends against fusion and damage. Human telomeres contain six nucleotide sequences repeated many times. The total length of telomeres is $10-20 \mathrm{kbp}$. They are called the biological clock of the cell, because telomeres shorten with every cell division. Extremely short telomeres are a signal to induce programmed cell death [4]. Various factors may affect the length of telomeres, such as drugs, inflammation or unhealthy diet [5]. Faster telomere shortening is associated with a higher risk of developing certain diseases, such as diabetes, atherosclerosis and cancers [6-8]. The results of published studies show that telomeres are a promising molecular marker that can be useful in predicting the risk of diseases. As mentioned above, telomeres in all somatic cells are shortened with age. The same situation is observed in female reproductive cells, but in sperm cells, telomeres are extended with age [9]. There are only a few, and in some cases, divergent reports on telomere length in sperm cells. Some of them indicate that there is a correlation between telomere length, semen quality and fertilization capacity [3,9-14]. Recent studies have also reported that telomere length in sperm cells play an important role in early embryo development $[15,16]$. Fertilization with a sperm cell with short telomeres may decrease chances of implantation and normal embryo development, and may be associated with early pregnancy loss $[15,17]$. The aim of the study was to investigate whether there are any differences between telomere length in men of similar age with normal and abnormal semen parameters. 


\section{Materials and Methods}

\section{Ethical Approval}

The research project has been approved by the local Ethics Committee. Biological material was derived from patients, who gave semen for routine examination. All patients were informed about the aim of the study and each participant gave his written informed consent.

\section{Subjects}

Forty-five donors were divided into two groups: with normal and abnormal semen parameters. All donors were of reproductive age (Table 1).

Table 1: Basic characteristics of sperm cells donors.

\begin{tabular}{|c|c|c|c|}
\hline Group of Patients & Number of Donors & [\%] of Donors & Age (mean \pm SD) \\
\hline All patients & 45 & 100 & $31,8 \pm 4,4$ \\
\hline Normozoospermia & 17 & 38 & $32 \pm 4,2$ \\
\hline Men with lower fertility & 28 & 62 & $31,6 \pm 4,5$ \\
Asthenozoospermia & 3 & 7 & $33 \pm 3$ \\
Astheno Teratozoospermia & 12 & 27 & $29,7 \pm 4$ \\
Oligo Astheno Teratozoospermia & 7 & 15 & $33 \pm 4,9$ \\
Teratozoospermia & 6 & 13 & $33 \pm 5,1$ \\
\hline
\end{tabular}

\section{Sperm Cell Preparation}

Sperm cells were prepared by density gradient centrifugation. It was important to separate spermatozoa from non-germ cells and other debris. About $1.5 \mathrm{ml}$ of fresh liquefied semen was layered on top of $80 \%$ gradient (Pureception $80 \%$ Lower Phase solution, SAGE) and centrifuged at $600 \mathrm{~g}$ for 15 minutes. The pellet was resuspended in $2 \mathrm{ml}$ of equilibrated medium for further washing after removing the gradient layer and seminal fluid, without disturbing the sperm pellet, (Flushing Medium, ORIGIO) and centrifuged at $600 \mathrm{~g}$ for 5 minutes. In the last step, the supernatant was removed and the pellets were resuspended in a suitable volume of washing medium.

\section{Genomic DNA Extraction}

Genomic DNA was extracted directly from sperm cells using a Macherey-Nagel kit (NucleoSpin Tissue) with minor modifications. Approximately $150 \mu \mathrm{l}$ of semen was diluted in GuEX buffer $(2 \mathrm{ml}$, $5 \mathrm{M}$ guanidine hydrochloride, $2,1 \mathrm{ml}$ Tris- $\mathrm{HCl}, 1,05 \mathrm{ml} 2 \mathrm{M} \mathrm{NaCl}$, $4,2 \mathrm{ml} 0,5 \mathrm{M}$ EDTA, $0,2 \mathrm{ml} 1 \mathrm{M} \mathrm{NaOH}, 190 \mathrm{ml}$ of water) and isolated according to the manufacturer's instructions.

\section{Quantitative Polymerase Chain Reaction (QPCR)}

Table 2: Sequence of primers and oligomers used in qPCR.

\begin{tabular}{|c|r|c|}
\hline $\begin{array}{c}\text { Primer/ } \\
\text { oligomer }\end{array}$ & Sequence \\
\hline Primer Telg & ACACTAAGGTTTGGGTTTGGGTTTGGGTTTGGGTTAGTGT \\
\hline Primer Telc & TGTTAGGTATCCCTATCCCTATCCCTATCCCTATCCCTAACA \\
\hline Primer Albu & CGGCGGCGGGCGGCGCGGGCTGGGCGGaaatgctgcacagaatccttg \\
\hline Primer Albd & GCCCGGCCCGCCGCGCCCGTCCCGCCGgaaagcatggtcgcctgtt \\
\hline Oligomer tel & TTAGGGTTAGGGTTAGGGTTAGGGTTAGGGTTAGGGTTAGGGTTAGGGTTAGGGTTAGGGTTAGGGTTAGGGTTAGGG & {$[18]$} \\
\hline Oligomer alb & CAGAGTCACCAAATGCTGCACAGAATCCTTGGTGAACAGGCGACCATGCTTTTCAGCTCTGGAA \\
\hline
\end{tabular}

Telomere length was determined using monochrome multiplex quantitative PCR (MMQPCR), according to Cawthon [18] with modifications [19]. The dilution series (100pg to $0.0001 \mathrm{pg}$ for telomere oligomer and $1 \mathrm{pg}$ to $0.0001 \mathrm{pg}$ for albumine oligomer) of synthetic oligomers was used as a reference sample to prepare the standard curve (Table 2). Two standard curves were prepared for each plate, one for telomeres and the other for the reference gene, i.e., albumin. All experimental and standard samples were run in triplicate. Each reaction well contained $2 \mu$ of DNA (10ng), 2x SYBR Green PCR Master Mix (Roche), two pairs of primers (telg and telc primer-500nM; albu and albd primer-900nM) and water to a final volume of $10 \mu$ l. Thermal cycling profile was as follows: $15 \mathrm{~min}$ at $95^{\circ} \mathrm{C}, 2$ cycles of $15 \mathrm{~s}$ at $94^{\circ} \mathrm{C}, 15 \mathrm{~s}$ at $49^{\circ} \mathrm{C} ; 35$ cycles of $15 \mathrm{~s}$ at $94^{\circ} \mathrm{C}, 10 \mathrm{~s}$ at $62^{\circ} \mathrm{C}, 15 \mathrm{~s}$ at $74^{\circ} \mathrm{C}$ with signal acquisition, $10 \mathrm{~s}$ at $84^{\circ} \mathrm{C}, 15 \mathrm{~s}$ at $88^{\circ} \mathrm{C}$ with signal acquisition. After thermal cycling and raw data collection, CFX Manager Software (Bio-Rad) was used to generate two standard curves for each plate. The efficiency of the reaction was equal for telomeres and albumine and it was not 
lower than 95\%. The variation of $\mathrm{Ct}$ values in the sample was $<0.5$ $\mathrm{Ct}(\mathrm{SD}<0.25)$ in both telomere and albumine runs. Mean Ct values were used to calculate absolute telomere length in kbp per haploid genome, as described by O'Callaghan [19]. Statistical analysis. The normality of the variables was evaluated by the Shapiro-Wilk test. The association of telomere length and semen parameters, such as concentration, vitality and mobility was analyzed with Spearman rank correlation coefficient (rs). P-values lower than 0.05 were considered as significant. Statistical differences in telomere length between two groups: donors with normozoospermia and donors with lower fertility were analyzed by Student's t-test. Data are reported as mean $\pm \mathrm{SD}$.

\section{Results}

Q-PCR quality control showed that the slope of the standard curve for both telomere and albumine reaction was-3.323 and-3.338, and the values of the acceptable liner correlation coefficient $\left(R^{2}\right)$ for both reactions were 0.997 and 0.997 , respectively. The efficiency of Q-PCR for telomere and albumine was $100.0 \%$ and $99.3 \%$, respectively (Figure 1) \& (Table 3) shows basic characteristics of semen parameters in all donors. Mean sperm telomere length in all donors was $49 \pm 24 \mathrm{kbp}$ per haploid genome. However, patients with abnormal semen parameters had significantly longer STL when compared with patients with normal semen parameters $(55 \pm 28 \mathrm{kbp}$ vs $38 \pm 12 \mathrm{kbp}$ ). Higher STL variability was observed in patients with abnormal semen parameters compared to patients with normozoospermia (Figure 2). Among standard semen parameters, Sperm Telomere Length (STL) in patients with normozoospermia was positively associated with sperm concentration $(\mathrm{P}=0.006)$ and vitality ( $\mathrm{P}=0.01$ ) (Figure 3). There was no correlation between STL and motility in those donor group. Negative correlation between STL and vitality $(\mathrm{P}=0.032)$ and sperm concentration $(\mathrm{P}=0.011)$ was observed in donors with abnormal semen parameters (Figure 4). There was no correlation between donor age and STL.

Table 3: Basic characteristic and semen parameters of all donors.

\begin{tabular}{|c|c|c|c|}
\hline Group of Patients & Concentration (mln/ejaculate) & Sperm Vitality (\%) & Progressive Motility (\%) \\
\hline All donors & $259 \pm 260$ & $74,7 \pm 12$ & $34 \pm 20$ \\
\hline Normozoospermia & $436 \pm 267$ & $80 \pm 6$ & $23,5 \pm 13$ \\
\hline Patients with lower fertility & $151 \pm 188$ & $71 \pm 13$ & $20 \pm 7$ \\
\hline Asthenozoospermia & $136 \pm 70$ & $73 \pm 7$ & $17 \pm 10$ \\
\hline AsthenoTeratozoospermia & $194 \pm 230$ & $70,5 \pm 13,8$ & $15,8 \pm 8,8$ \\
\hline OligoAsthenoTeratozoosperma & $13,9 \pm 9$ & $65,8 \pm 17,6$ & $46,6 \pm 9,2$ \\
\hline Teratozoospermia & $235 \pm 186$ & $79,6 \pm 6$ & 2 \\
\hline
\end{tabular}
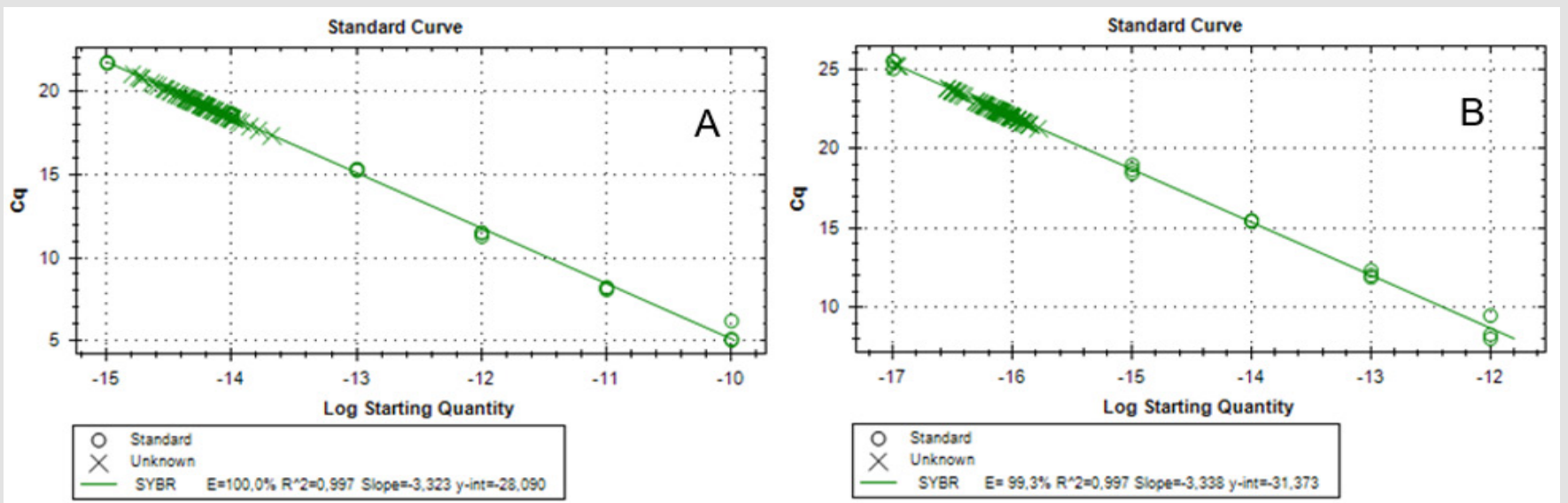

Figure 1: Standard curves for telomeres (A) and for albumine (B). 


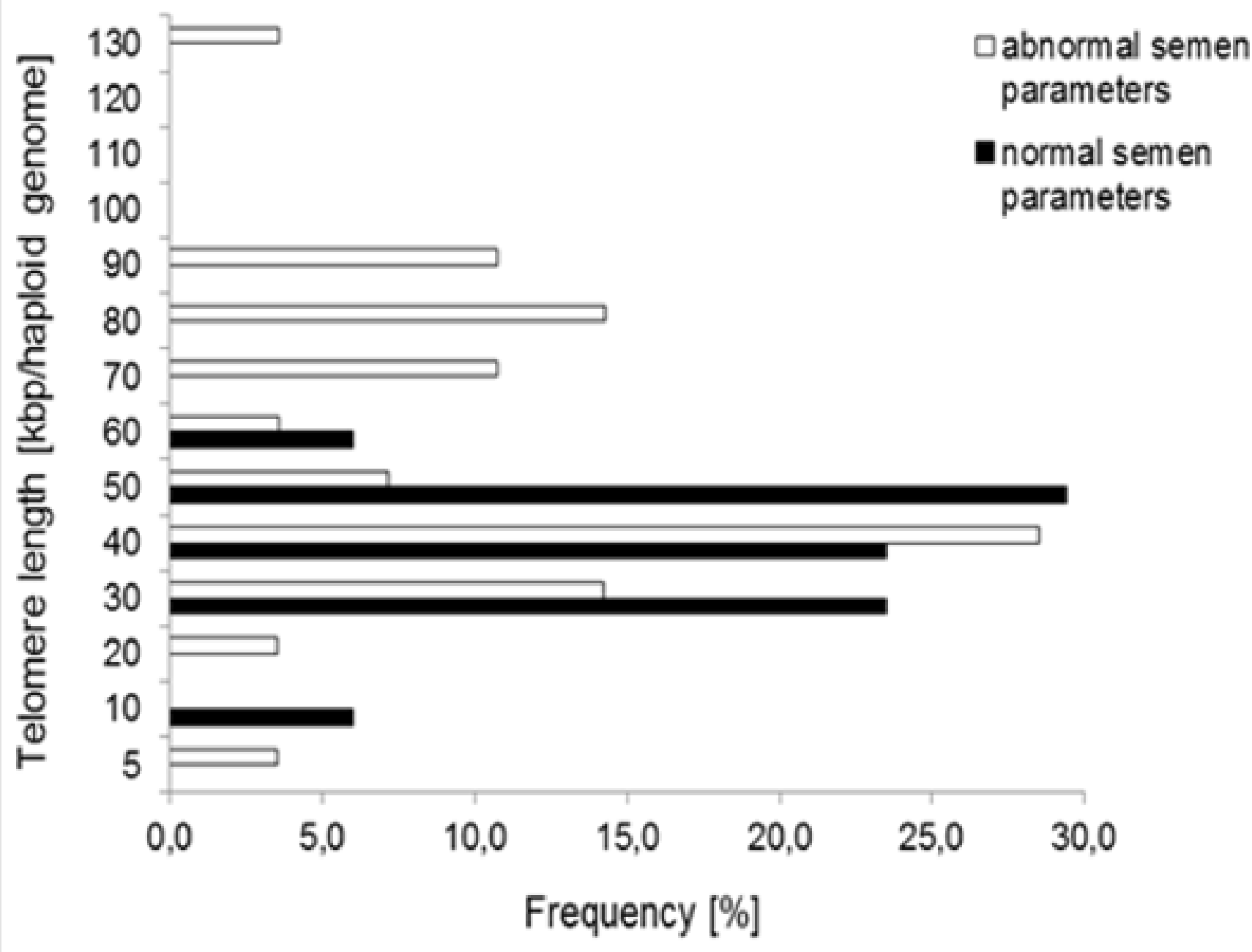

Figure 2: Sperm telomere length of patients with normal and abnormal semen parameters.
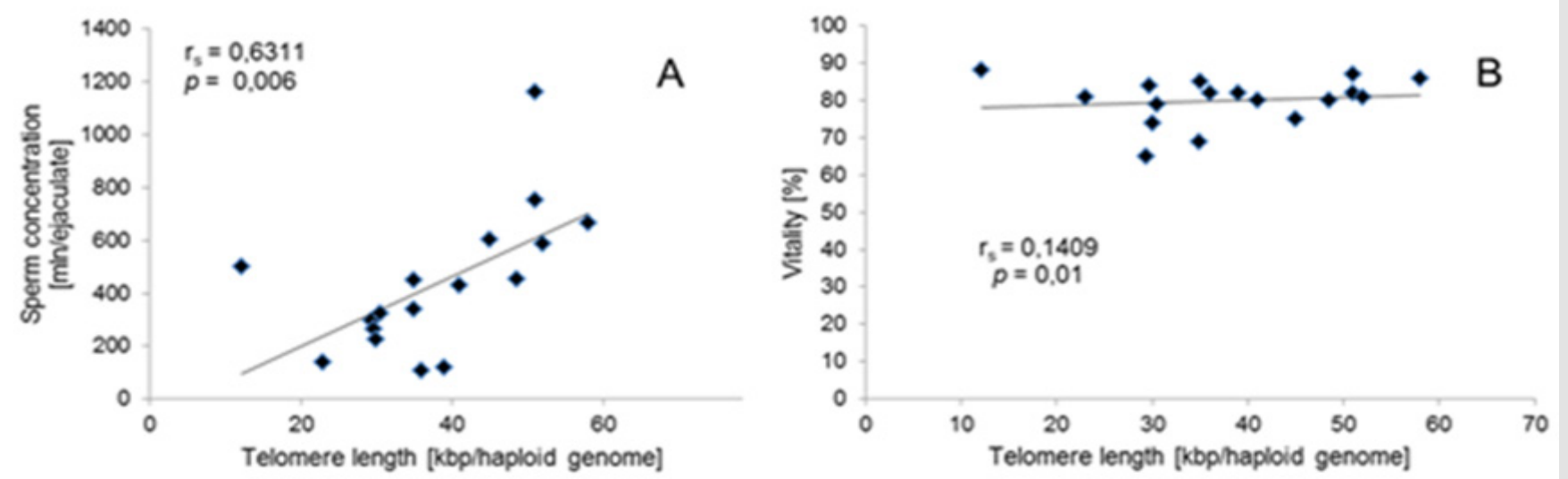

Figure 3: Associations for sperm telomere length of patients with normal semen parameters. (A) Correlation between sperm concentration and Sperm Telomere Length (STL). (B) Correlation between STL and vitality. 


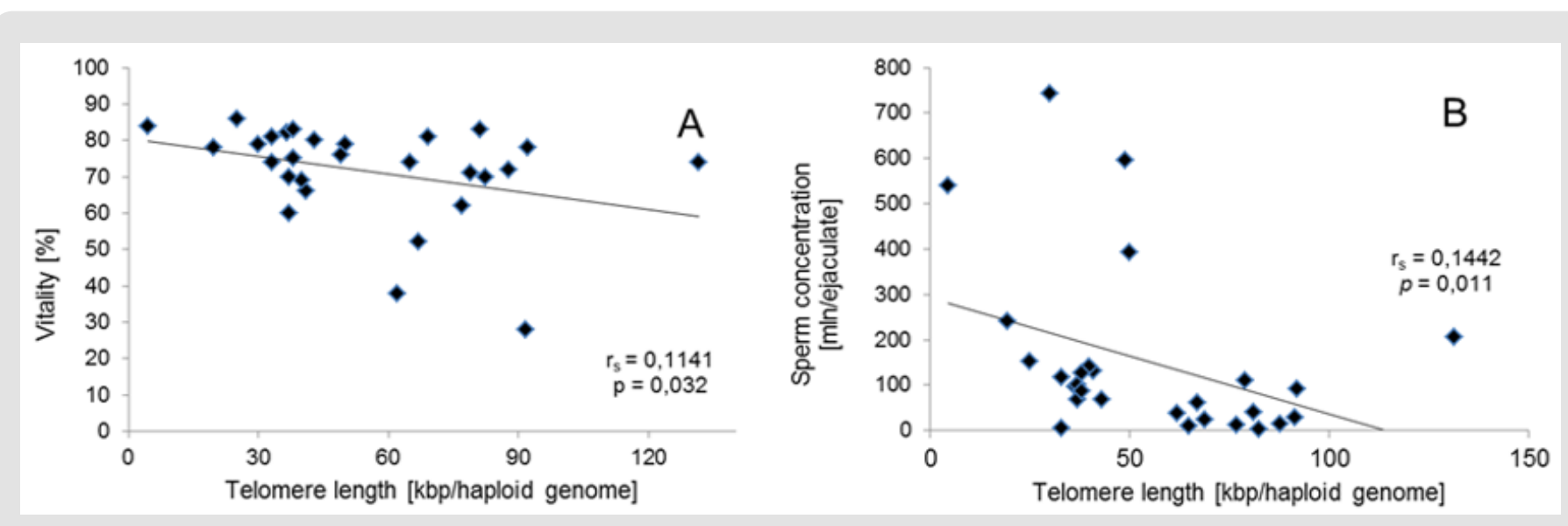

Figure 4: Associations for sperm telomere length of patients with abnormal semen parameters. Correlation between vitality and sperm telomere length. (B) Correlation between STL and sperm concentration.

\section{Discussion}

Telomere length and structure play an important role in maintaining genome integrity and they are also crucial for recombination, meiosis and pronucleus formation, following the entry of the sperm into the oocyte. The role of sperm telomere length in male fertility is not known [3]. As indicated by Kimura et al. [20], mean STL in sperm does not decrease. In fact, it increases with donor age. Older men have longer telomeres. Fortyfive sperm donors of similar age were examined. Seventeen of them were characterized by normozoospermia. The rest of them had reduced fertility. In addition, this group included men with different, reduced sperm parameters (Tables 1-3). In our study, patients with normozoospermia had lower telomeres than patients with abnormal semen parameters (Figure 2). Recent studies by Rocca et al. $[14,11]$ demonstrated that STL increased with age and men with oligozoospermia had shorter sperm telomeres than normozoospermic men.

Shorter telomeres in sperm may be one of the causative factors responsible for male infertility, but further detailed studies are needed to confirm these findings [3]. The results of scientific studies on telomere length in infertile men concern patients with oligozoospermia and idiopathic infertility [21]. In our study group, there are patients with changes in sperm motility and morphology. Perhaps telomere length is different in these study groups compared with patients with oligozoospermia and idiopathic infertility. More research is needed to prove this hypothesis. Moreover, we have not taken into account other factors like lifestyle, oxidative stress and environmental pollution. Mild oxidative stress results in telomere lengthening, but severe oxidative stress causes shorter telomeres [22]. Although telomere maintenance is a complex trait, studies have shown that mild oxidative stress is beneficial in maintaining telomere length, and thus a delicate balance needs to be established to maximize the beneficial effects of free radicals and prevent harmful effects of supra-physiological levels [22-24]. Oxidative stress induces an inverse relationship between telomere length and abnormal nuclear morphologies [25]. The another studies indicate that male residents in areas with high environment exposure had a significant increase in STL. This finding supports the view that the human semen is a sentinel biomarker of environmental exposure [26]. Acute and chronic exposure to high levels of ambient pollutants may impair male fertility by affecting sperm quality, but the real effect size remains controversial [14,27] reported that STL was associated with standard semen quality parameters and, more importantly, it was significantly associated with the level of DNA fragmentation and sperm protamination. Among standard semen parameters, STL was positively associated with progressive motility and vitality.

In our study, STL in patients with normozoospermia was positively associated with sperm concentration and vitality. There was no correlation between STL and motility in these donor groups. Negative correlation between STL and vitality and sperm concentration was observed in donors with abnormal semen parameters. Unlike the latter study, we found no correlation between STL and age, as in other [11-30]. However, for the present study, we selected men with normozoospermia as control and men with astenozoospermia, teratozoospermia, astenoteratozoospermia and oligoastenoteratozoospermia in the same age range. This is probably the reason why the aforementioned associations were not observed and STL in patients with normozoospermia was lower than in patients with abnormal semen parameters. In fact, the demonstration that STL is an additional sperm parameter may open new perspectives in the evaluation of infertile males [31,32]. Additional research on men of proven fertility and infertility will clarify the significance of this biomarker also as a quality parameter for semen.

\section{Conclusion}

Patients with abnormal semen parameters had significantly longer STL in comparison to patients with normal semen parameters. If tests in other patients confirm these results, telomere length can be considered as a promising molecular marker of male 
fertility in sperm donors with abnormal sperm morphology and motility.

\section{Acknowledgement}

The study was supported by internal grant from Department of Biotechnology University of Opole. The authors thank Natasha $\mathrm{Ng}$, a scholarship holder of the Fulbright Foundation for linguistic correction of the text.

\section{Conflicts of Interest}

The authors declare that they have no conflicts of interests

\section{References}

1. Jungwirth A, Diemer T, Dohle GR, Kopa Z, Krausz H, et al. (2015) Guidelines on Male Infertility. European Association of Urology.

2. (2015) Practice committee of the american society for reproductive medicine diagnostic evaluation of the infertile male: A committee opinion. Fertil Steril 103:18-25.

3. Thilagavathi J, Kumar M, Mishra SS, Venkatesh S, Kumar R, et al. (2013) Analysis of sperm telomere length in men with idiopathic infertility. Arch Gynecol Obstet 287: 803-807.

4. Sosińska P, Pietrasik MJ, Książek K (2016) Molecular bases of cellular senescence: Hayflick phenomenon 50 years later. Postepy Hig Med Dosw 70: 231-242.

5. Shammas MA (2011) Telomeres, lifestyle, cancer and aging. Curr Opin Clin Nutr Metab Care 14: 28-34.

6. Chen S, Lin J, Matsuguchi T, Blackburn E, Yeh F, et al. (2014) Short leukocyte telomere length predicts incidence and progression of carotid atherosclerosis in american indians: The strong heart family study. Aging (Albany NY) 6: 414-427.

7. Willeit P, Raschenberger J, Heydon EE, Tsimikas S, Haun M, et al. (2014) Leucocyte telomere length and risk of type 2 diabetes mellitus: New prospective cohort study and literature-based meta-analysis. PLoS One 9: 112483.

8. Hou L, Blaser MJ, Perez-Perez G, Hoxha M, Dioni L, et al. (2009) Telomere length in peripheral leukocyte DNA and gastric cancer risk. Cancer Epidemiol Biomarkers Prev 18(11): 3103-3109.

9. Turner S, Hartshorne GM (2013) Telomere lengths in human pronuclei, oocytes and spermatozoa. Mol Hum Reprod 19(8): 510-518.

10. Antunes DM, Kalmbach KH, Wang F, Dracxler RC, Seth-Smith ML, et al. (2015) A single-cell assay for telomere DNA content shows increasing telomere length heterogeneity, as well as increasing mean telomere length in human spermatozoa with advancing age. J Assist Reprod Genet 2: $1685-1690$.

11. Ferlin A, Rampazzo E, Rocca MS, Keppel S, Frigo AC, et al. (2013) In young men sperm telomere length is related to sperm number and parental age. Hum Reprod 28: 3370-3376.

12. Shuyuan L, Changjun Z, Haiying P, Xiaoqin H, Hao S, et al. (2015) Association study of telomere length with idiopathic male infertility. Yi Chuan 37: 1137-1142.

13. Mishra SS, Kumar S, Singh G, Mohanty K, vaid S, et al. (2015) Oxidative DNA damage in male germ cells in normozoospermic infertile men: A case for concern. Austin J Reprod Med Infertil 2:1017.
14. Rocca MS, Speltra E, Menegazzo M, Garolla A, Foresta C, et al (2016) Sperm telomere length as a parameter of sperm quality in normozoospermic men. Hum Reprod 1: 1158-1163.

15. De Frutos C, López-Cardona AP, Fonseca Balvís N, Laguna-Barraza R, Rizos D, et al. (2016) Spermatozoa telomeres determine telomere length in early embryos and offspring. Reprod 151(1): 1-7.

16. Yang Q, Zhao F, Dai S, Zhang N, Zhao W, et al. (2015) Sperm telomere length is positively associated with the quality of early embryonic development. Hum Reprod 30(8): 1876-1881.

17. Thilagavathi J, Mishra SS, Kumar M, Vemprala K, Deka D, et al. (2013) Analysis of telomere length in couples experiencing idiopathic recurrent pregnancy loss. J Assist Reprod Genet 30: 793-798.

18. Cawthon R (2009) Telomere measurement by the novel monochrome multiplex quantative PCR method. Nucleic Acids Res 37: 21-28.

19. O'Callaghan N, Fenech M (2011) A quantitative PCR method for measuring absolute telomere length. Biol Proced Online 13: 3.

20. Kimura M, Cheras LF, Kato B, Demissie S, Hjelmborg JB, et al. (2008) Offspring's leukocyte telomere length, parental ages, and telomere elongation in sperm. PloS Genet 4: 37.

21. Cariati F, Jaroudi S, Alfarawati S, Raberi A, Alviggi C, et al. (2016) Investigation of sperm telomere length as a potential marker of paternal genome integrity and semen quality. Reprod Biomed Online 33(3): 404411.

22. Mishra SS, Kumar R, Malhotra N, Singh N, Dada R, et al. (2016) Mild oxidative stress is beneficial for sperm telomere length maintenance. World J Method 6(2): 163-170.

23. Kawanishi S, Oikawa S (2004) Mechanism of telomere shortening by oxidative stress. Ann NY Acad Sci 1019: 278-284.

24. Wang W, Chen H, Li R, Ouyang N, Chen J, et al. (2004) Telomerase activity is more significant for predicting the outcome of IVF treatment than telomere length in granulosa cells. Reprod 147: 649-657.

25. Coluzzi E, Colamartino M, Cozzi R, Leone S, Meneghini C, et al. (2014) Oxidative stress induces persistent telomeric DNA damage responsible for nuclear morphology change in mammalian cells. PLoS One 9: 110963.

26. Vecoli C, Montano L, Borghini A, Notari T, Guglielmino A, et al. (2017) Effects of highly polluted environment on sperm telomere length: A pilot study. Int J Mol Sci 18(8): pp. E1703.

27. Deng Z, Chen F, Zhang M, Lan L, Qiao Z, et al. (2016) Association between air pollution and sperm quality: A systematic review and meta-analysis. Environ Pollut 208(Pt B): 663-669.

28. Allsopp RC, Vaziri H, Patterson C, GoldStein S, Younglai EV, et al. (1992) Telomere length predicts replicative capacity of human fibroblasts. Proc Natl Acad Sci USA 89(21): 10114-10118.

29. Aston KI, Hunt SC, Susser E, Kimura S, Factor-Litvak P, et al. (2012) Divergence of sperm and leukocyte age-dependent telomere dynamics: Implications for male-driven evolution of telomere length in humans. Mol Hum Reprod 18(11): 517-522.

30. Baird DM, Britt-Compton B, Rowson J, Amso NN, Gregory L, et al. (2006) Telomere instability in the male germline. Hum Mol Genet 15: 45-51.

31. Boniewska-Bernacka E, Pańczyszyn A, Cybulska N (2018) Telomeres as a molecular marker of male infertility. Human Fertility 22(2): 78-87.

32. Rocca MS, Foresta C, Ferlin A (2018) Telomere length: Lights and shadows on their role in human reproduction. Biol Reprod. 


\section{ISSN: 2574-1241}

DOI: 10.26717/BJSTR.2019.21.003559

Ewa Boniewska-Bernacka. Biomed J Sci \& Tech Res

cC (P) This work is licensed under Creative BY Commons Attribution 4.0 License

Submission Link: https://biomedres.us/submit-manuscript.php

\begin{tabular}{ll} 
BIOMEDICAL & \multicolumn{1}{c}{ Assets of Publishing with us } \\
RESEARCHES & - Global archiving of articles \\
& - Immediate, unrestricted online access \\
& - Rigorous Peer Review Process \\
\end{tabular}

